\title{
A LAYERED APPROACH TO WEARABLE TEXTILE NETWORKS
}

\author{
Kristof Van Laerhoven, Nicolas Villar and Hans-Werner Gellersen \\ Department of Computing \\ Lancaster University \\ LA14YR Lancaster, United Kingdom \\ \{kristof, villar, hwg\}@comp.lancs.ac.uk
}

\begin{abstract}
The integration of digital components into clothing is becoming an increasingly important segment in wearable computing research. The first indications for this trend are the incorporation of existing mobile technologies, such as personal digital assistants (PDAs) or mobile phones, into jackets via flexible textile circuits. In the long term, other components could also be envisioned that are embedded in apparel, using a flexible bus-type network that links all the devices together. This paper introduces a functioning prototype of such a flexible network that not only allows communication between wearable components, but is also able to supply power to them. We propose an arrangement of layered textiles as opposed to the more traditional routed circuitry layout, which results in a novel approach towards the concept of a flexible clothing network.
\end{abstract}

\section{INTRODUCTION}

Progress in coating and weaving techniques has enabled industry and research to produce durable threads and textiles that are highly conductive and as flexible as regular fabric. Research at MIT (5), France Telecom (6), Foster-Miller (3), Reima Smart Clothing (12) and Philips $(2,4)$ has produced products that use this conductive textile to network the wearer's mobile devices (such as an MP3 player or a mobile phone). As these devices get smaller in size and larger in variety, it is plausible that the importance of this flexible clothing network will increase.

The routing of power and communication paths in clothing is commonly achieved by weaving a number of conductive threads along predefined seams, similar in structure to how peripheral devices are connected to a personal computer. The locations where these wearable devices can be stored are therefore limited to the area around these routes, assuming these are fixed in the clothing. Using this integrated routed approach can therefore become more awkward in applications where the accurate location of these devices is essential.

Research in wearable sensor networks in particular, requires the interconnection of a large number of sensor devices that can be worn at certain places on the body. Experiments on prototypes with conventional cables have proven to be inferior, as they hinder the wearer's movements and tend to put a lot of strain on the connectors. The sheer weight of the cabling, as well as the space that they require, rapidly increases as more sensors are added to the clothing network.

This paper will present a structural design method for the physical integration of network connections in clothing, which is different from traditional techniques. After an overview of how the network functions and what its components are, a toy problem illustrates the implementation, benefits and prospects of this type of networking in wearable computing. This is followed by a discussion on the approach's limitations and application potential.

\section{A LAYERED DESIGN}

This paper focuses on the use of layers instead of routes, to carry data and power over clothing. This expands the network over flexible two-dimensional planes rather than wires, and results in the distribution of the network over the entire surface of the garment. Moreover, using conducting layers also does away with the need for fixed routing.

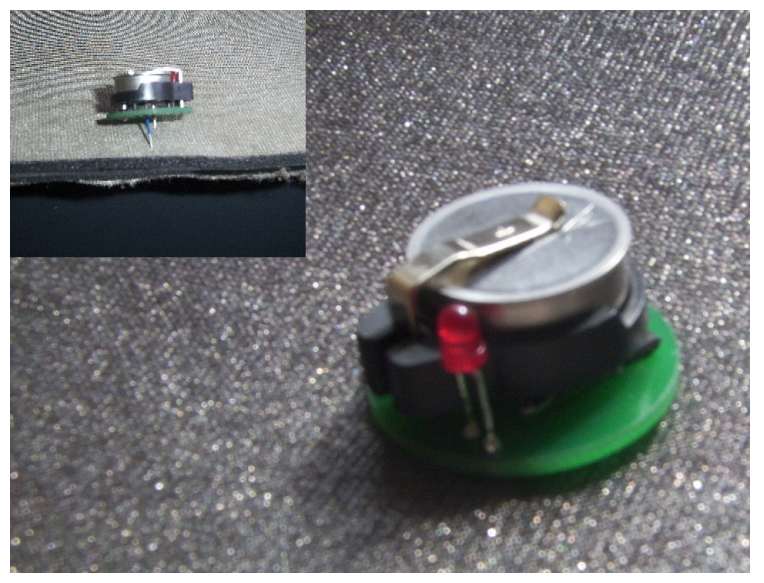

Figure 1. Pictures of the proposed layered textile network, where two conductive layers are separated by a rubber layer, and a wearable device. The network connection is made by attaching the device like a lapel pin. 
To characterize this approach, an example is given specifying the network bus protocol, the physical arrangement of the layers, and the method of connecting the networked devices to the separate layers.

\section{The Network Protocol}

The protocol that has been adopted in this paper is responsible for providing both a communication network and power to any device that gets connected. The Dallas MicroLAN (7) is a protocol standard that needs two connections to network and power devices: one for ground, and one for the communication bus' signal. The latter signal is furthermore pulled to a high state most of the time, so that the attached devices are able to 'steal' power from that same communications bus.

The traffic on the bus network is controlled on a master-slave principle, where one node controls all messages from the other nodes, dubbed slaves or clients. The complexity of this master node depends on the specific network application, but can be implemented in embedded devices.

\section{The Physical Network Materials}

The conductive textile that was used in this paper consists of woven threads of nylon that have been coated with silver (AG) to give it conductive properties. The layers of this fabric are insulated by flexible sheets of rubber that is available with adhesive sides that make it easier to secure to the conductive layers. These two materials are widely available, relatively low-cost, and robust enough to be used in clothing.

The silver-coated fabric was chosen for its high conductivity ( 0.5 Ohms per square), but other coatings are possible as well (such as Copper) (8). Alternatively, one can also use actual fibers of conductive alloys rather than coating that are then used in a woven or knitted fabric (1). Both types of fabrics (using coated nylon or metal fibers) are generally used in radio frequency shielding and anti-static applications.

\section{CASE STUDY: TEMPERATURE LOGGING}

The case study in this paper uses the network described in the previous section to solve a model problem of a wearable sensor network: Consider that one would like to know the temperature distribution over the entire area of a piece of clothing while it is being worn, over a certain period (covering hours, days, or possibly even months).
Consider also certain additional restrictions on the system:

- For increasing its robustness, there might be the need to log the temperature data locally in the sensor modules themselves.

- There is furthermore a need for flexibility in the network: it should be straightforward to add, reposition, or replace sensors at any time.

\section{The Sensor Modules}

Having described the network, both physically and in software protocol in the previous section, the only remaining component that needs to be specified is the sensor module that carries out the measuring and logging of the temperatures. We use for this purpose the DS1921H iButton device, or Termochron (9), which also interoperates with the MicroLAN network communication standard (as all iButton devices do).

The Termochron records both time and temperature, it's embedded computer chip integrates a MicroLAN transmitter/receiver, a globally unique address, a high resolution thermometer, a clock/calendar, a thermal history log, and 512 bytes of additional memory to store additional data. It comes also integrated with a $3 \mathrm{~V}$ battery that keeps its logging process running for more than 10 years, for temperatures from $15^{\circ} \mathrm{C}$ to $46^{\circ} \mathrm{C}$, with a $\pm 0.125^{\circ} \mathrm{C}$ accuracy. Each iButton also has an ID number that is unique in the world, formed by 64 bits or 8 bytes.

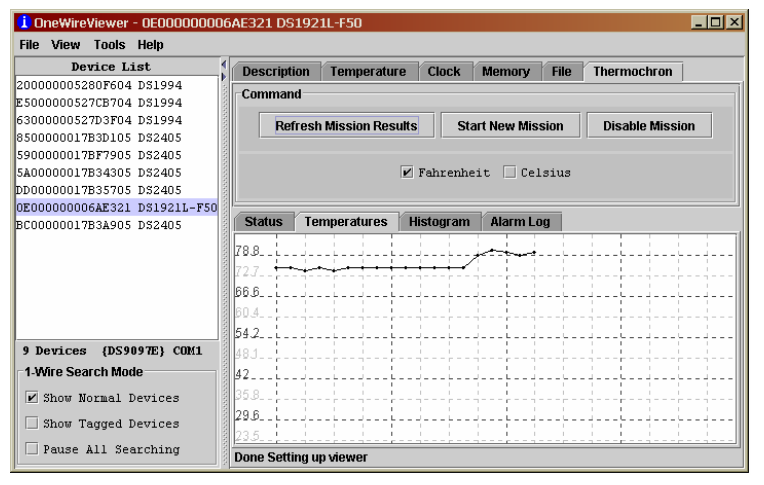

Figure 2. Screenshot of the OneWireViewer utility that comes with MicroLAN parts. The IDs of the devices present on the bus network are listed on the left, while device specific information (such as the logged temperatures for the Thermochron) is displayed on the right.

As mentioned in the protocol section, MicroLAN devices typically communicate with a bus master controller. Adaptors are available that convert the 
signals from the MicroLAN to serial RS232 levels so that the network can be 'inspected' by software on a $\mathrm{PC}$, as Figure 2 illustrates.

\section{The Connection}

The temperature loggers still need to be capable of ad-hoc connection to the physical network. For this reason, the temperature logging iButtons are fitted with two pins: one short pin that connects to the closest layer, and one partly-insulated longer one that connects to the second layer. In this fashion, all 'pinned' modules access the same two layers that provide the network connections (see Figure 3 for a diagram).

Based upon earlier work from the Pin\&Play $(10,11)$ project that uses similar hardware and materials for creating wall-sized networks, we arrive at a flexible, textile-based network, connecting small devices that do distributed sensing.

Using these pin connectors, the small temperature modules can thus be seen as an electronic enhancement of lapel pins that traditionally get attached to lapels or ties. Typically, these pins come with a clip to protect the wearer from the sharp pin (Figure 3 provides a photograph of an example of a decorative lapel pin).

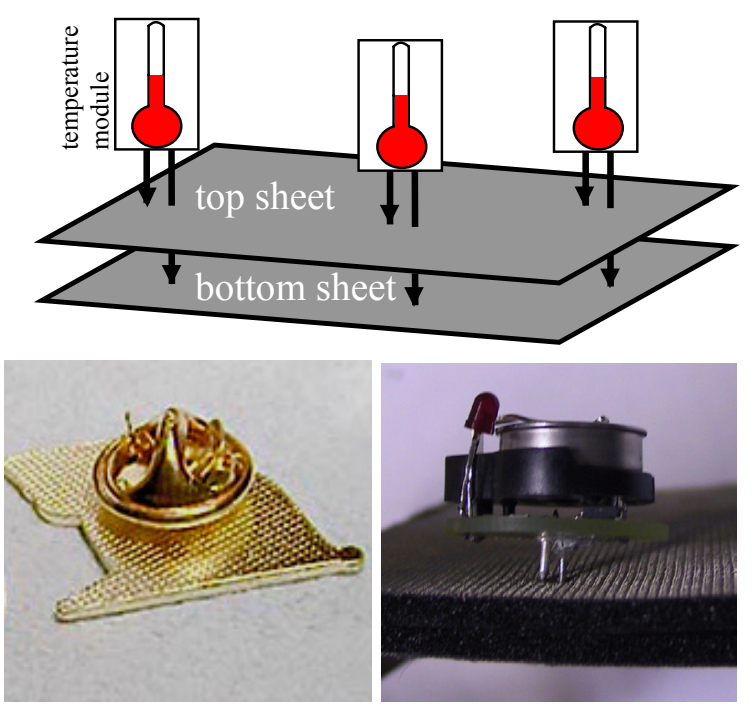

Figure 3. Schematic of the network (top), a photograph of an ordinary lapel pin (bottom left), and this paper's implemented prototype pin and network (bottom right). Temperature logging modules can thus be added and replaced on the fabric, similar to lapel pins. These augmented pins however contain an additional temperature sensor, data logger, and MicroLAN communication capabilities.

\section{EXPERIMENT}

This section aims to demonstrate the feasibility of using the prototype hardware discussed in the previous sections, in particular for the introduced wearable sensing problem. A further goal is to characterize the performance of the proposed network using this example.

As temperature logging is completely the responsibility of the Thermochron iButtons, the software for this application that runs on the master comes into action after all the data has been stored. All components use their own internal battery power while the experimental garment is worn, and no communication between nodes is necessary. The network master (a standard desktop PC in this experiment) therefore does not have to be connected until after the trial-period, when all the devices need to deliver their logged temperature data.

The advantage of using the layered approach to garment construction in this case study is the flexibility of location of the sensing devices on the jacket. Should the experimenters want to redo the trial with additional sensors, or sensors on other locations, all that needs to be done is to simply attach or re-attach the devices, respectively. No new wires need to be rerouted in the jacket, and no changes in software or hardware are required.

The network protocol used (MicroLAN) comes from a traditional wired bus standard. Our layered network variation therefore does not provide the location where on the body a device is located, so this information has to be assigned by hand. Once this is done, however, all other information (mainly consisting out of time-temperature data tupels) is downloaded and visualized instantaneously.

\section{Results}

In our experiments, the jacket was connected to a computer as soon as the trial phase was over (i.e., after sufficient temperatures had been logged), with the trial simply involving the jacket being worn for about 20 minutes (without the wearer doing anything) as a sanity check using the effects of body-heat. The only user-interaction that is required on the computer side at that point, is to input the positions of all temperature loggers. It would also have been feasible to connect a wearable computer to the jacket while the data logging was going on. However, the jacket is still in an early prototypical stage, which was the main reason for adopting this two-step process. 
To access the data from the temperature loggers, two types of response-times are of interest from an application-oriented perspective: the time to detect all devices is important for the responsiveness of the software, while the time to transmit the logged data is important for future applications (that might ask continuously for sensor data from all sensor modules across the clothing network, for instance).

The MicroLAN has in its protocol a built-in resetpresence-pulse procedure that was designed to quickly detect and identify all devices on the network. This explains the relatively high rates we measured between opening the network connection (between master and slaves), and the master having recorded all identities of the slaves. Table 1's 'Time To Read' column shows our application's response times, being the actual time the user has to wait between a query and the network's reply (including visualization, etc.). These figures show clearly the negative effects of the MicroLAN's low bandwidth, which for some applications would be unacceptable.

TABLE 1 - The Experiment's Time Measurements

\begin{tabular}{|c|c|c|}
\hline \# devices & $\begin{array}{c}\text { Time To Detect } \\
\left(\mathbf{m s}^{*}\right)\end{array}$ & $\begin{array}{c}\text { Time To Read } \\
\left(\mathbf{m s}^{*}\right)\end{array}$ \\
\hline 2 & 74 & 5200 \\
\hline 4 & 149 & 5600 \\
\hline 6 & 224 & 6100 \\
\hline 8 & 298 & 6600 \\
\hline 10 & 368 & 6800 \\
\hline
\end{tabular}

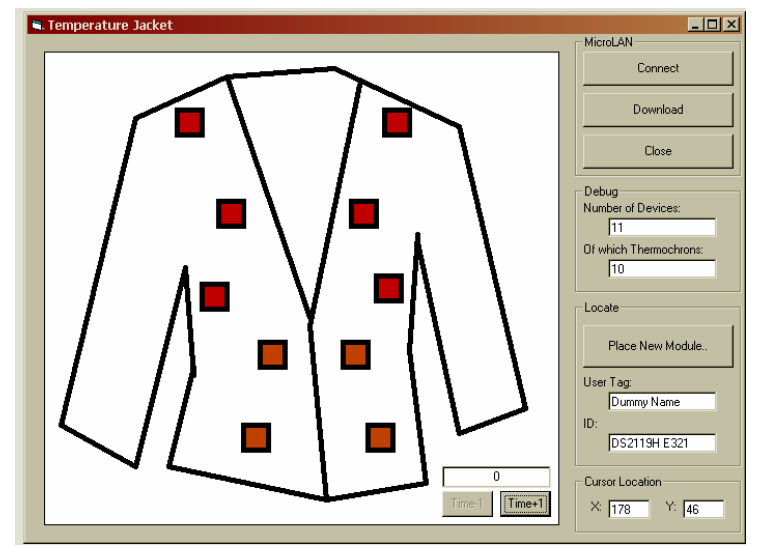

Figure 4. Screenshot of a final result: a mapping of temperatures over the jacket, showing squares where temperature sensors were located.

Figure 4 shows finally the outcome of a typical experiment, after the wearer has connected the jacket to the PC, and annotated the respective locations of the detected sensor devices. Figure 5 shows the prototype jacket's inner layer, containing several temperature logging devices, attached to a computer for analysis.

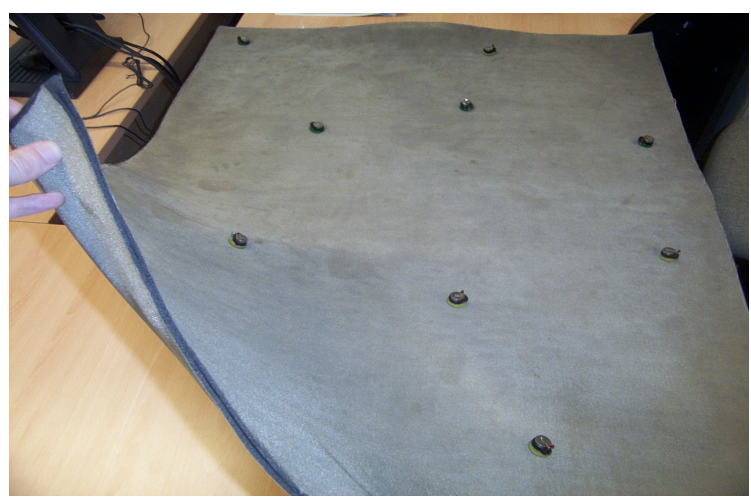

Figure 5. Picture of the prototype jacket's inside layer.

\section{DISCUSSION}

\section{Restrictions}

There are many reasons why the MicroLAN was chosen as a network protocol, with simplicity as the dominant factor. Since it needs only two channels, two insulated layers of conductive textile are sufficient, and the devices need only two pins to connect to those layers. But the MicroLAN comes also with some limitations: bandwidth is, at best, 16300 bits per second, and the network structure is limited to a master - slave architecture. This is sufficient for applications exchanging control packets, or short transmissions of data, but it would be incapable in its current form of supporting streams of video or sound.

A more physical disadvantage is the restriction on conductive pins: most normal lapel pins are fully conductive, so pinning them in the fabric layers would result in shorting the network. Both hardware and software can handle this kind of failure, but neither power nor communication will be available to the networked devices.

Finally, the layers of conductive fabric may interfere with wireless protocols operating in its vicinity. Initial tests with prototypes from the Pin\&Play project, however, indicate that the shielding effect is minimal as long as the wireless modules are not completely enclosed by the conductive layers (such as in a pocket). It is thus possible to use both layered and wireless approaches concurrently, given extra care is taken in positioning the antennae. 


\section{Layered vs. Wireless}

This paper proposes not only an alternative to routed networking in clothing; it obviously could also be an alternative to wireless networks. This section discusses possible reasons why certain applications might benefit from this paper's layered approach.

Power. Mobile and wearable computing devices are generally restricted in size and weight, which often leads to tough design choices on the batteries. Maintaining a wireless communication channel requires an additional amount of battery power, causing problems in the devices' design phase.

Size / Weight. Another prominent requirement for wearable computers is that their size and weight should not obstruct the wearer. The size of the pinbased devices is generally smaller, since they only need power to keep them working when they are detached from the network. They also do not require antennas or additional communication modules.

Network Range. Although some wearable devices may require connections with services in the surroundings of the wearer, most wearable devices for the same person have direct contact with his or her clothing. The network's signal strength and quality is also expected to remain the same.

Transparency of Network Topology. It is increasingly difficult to keep track of what devices are attached to one's network if it is wireless. The fact that devices have to be explicitly attached to the wearer's apparel in our proposed layered approach, makes it easier to get a prompt overview: Devices attached to the clothing, are attached to the network.

\section{CONCLUSIONS}

We proposed a new approach to integrate a physical network in clothing, using layers of conductive textile to bring power and communication capabilities to every square inch of the clothing's surface. Mobile and wearable devices can be attached to the fabric by pins that connect to each layer, thus giving the devices access to the clothing's power and communication network.

An application where a network is required to reach the clothing's entire surface was provided as a case study. It illustrated that the network can handle ad-hoc connections of many devices that were distributed over the clothing. The current prototype has, due to the chosen protocol, a low bandwidth as the key disadvantage. This, together with the more fundamental limitation that normal conductive lapel pins would disable the network, and an insight in health implications for this methodology, remain open issues.

The main advantage of spreading a network connection over the full surface of clothing is modularity: wearable devices are not required to have a fixed location or orientation on the clothing; they can be pinned and re-pinned at will, just like their traditional counterparts. Our case study showed that this property could be useful in specific areas such as wearable sensor research. The clothing-as-a-network paradigm has in our view potential beyond this domain, especially in longer-term wearable computing research.

\section{ACKNOWLEDGEMENTS}

This research was in part funded by the European Commission's IST FET framework, under contract code IST-2001-37007 (under project name: Pin\&Play), and by Equator, an EPSRC funded IRC project (GR/N15986/01 - "Technological Innovation in Physical and Digital Life"), as well as the EPSRC CommonSense project, under grant GR/S08848/01 ("Multi-Sensor Perceptive Interfaces in Wearable and Ubiquitous Computing").

\section{REFERENCES}

1. Bekaert Fibre Technologies (BFT) http://www.bekaert.com/bft/Products/Industrial \%20textiles/Metal\%20fibres\%20in\%20textiles. $\underline{\mathrm{htm}}$

2. Farringdon, J., Moore, A., Tilbury, N., Church, $J$. and Biemond P. (1999) Wearable Sensor Badge \& Sensor Jacket for Context Awareness. In Proceedings of the Third International Symposium on Wearable Computers, San Francisco, pp.107-113.

3. Foster-Miller Textile Networks: http://www.foster-miller.com/wearhome.html

4. Gough, P., Bickerton, M., Eves, D., Moore, A. and Massey, P. (2002). Wearable Electronics at Philips. Presentation at Eurowearable 2002.

5. Post, E. R., Orth, M., Russo, P. R. and Gershenfeld, N. (2000) E-broidery: Design and fabrication of textile-based computing . IBM Systems Journal, Volume 39, Numbers $3 \& 4$.

6. Mestaoui, N. and Aït Kaci, Y. (2002) Scenography accompanying the project 
"Echarpe communicante". http://www.electronicshadow.com/mediacol/sc eno/indexeng.htm

7. Dallas MicroLAN: Dallas Reference Manual MicroLAN Starter Kit, also known as DS9091K http://pdfserv.maxim-

ic.com/arpdf/DS9091K.pdf

8. Shieldex Coated Fabrics. http://www.shieldex.com

9. Dallas Thermochron $®$ iBButtons ${ }^{\circledR}:$ DS1921H Datasheet: http://pdfserv.maximic.com/arpdf/DS1921H-DS1921Z.pdf

10. Van Laerhoven, K., Villar, N., Schmidt, A. Gellersen, H.-W., Håkansson, $M$, and Holmquist, L. E. (2003) Pin\&Play: The Surface as Network Medium. IEEE Communications Magazine Vol.41 No.4. IEEE Press, pp. 90-96.

11. Van Laerhoven, K., Schmidt, A. Gellersen, H.W. (2002) Pin\&Play: Networking Objects through Pins. In Proc. of Ubicomp 2002, G. Boriello and L.E. Holmquist (Eds). Lecture Notes in Computer Science; Vol. 2498, Göteborg, Sweden. Springer Verlag, September 2002, pp.219 - 229. 